\title{
Long-Term Near-Ground Optical Scintillation Measurements
}

\author{
Dirk Giggenbach, Hennes Henniger, Florian David \\ German Aerospace Center, Oberpfaffenhofen, Germany
}

\begin{abstract}
A long-term measurement of the received-power fluctuations caused by atmospheric index-of-refraction turbulence in a near-ground optical free-space laser transmission experiment has been carried out over an eight-months period. Atmospheric index-of-refraction turbulence causes intensity variations in time and space (so called scintillations), these were monitored with several receiver apertures.

The purpose of this experiment was to gather data for the evaluation of atmospheric optical free-space transmission scenarios. These range from optical last-mile connections to interconnections of stratospheric platforms or satellite downlinks. The comparison with meteorological data conditionally allows predictions on other link scenario's behaviour under comparable boundary conditions. One motivation for this experiment was the prediction of free-space link performance based on easily measurable meteorological environmental conditions.
\end{abstract}

Keywords: Free-space laser communications, optical near-ground propagation, atmospheric turbulence, long-term scintillation measurements

\section{INTRODUCTION}

According to well-accepted theory the index of refraction of the atmosphere can be modelled as turbulence cells with different diameters and temperatures [1], [2], [3]. The turbulence itself is caused by heating of the air from the ground, convection-streams, and non-laminar wind movements. As the differential index of refraction is inverse proportionally related to the temperature of a gas, an optical wave passing through turbulent air will be distorted by the differently refracting volumes. This effect can be observed in an extreme case as the glimmering of the picture over a heated paved road. As the distorted wave propagates further it will get diffracted through the usual wave-propagation process and thus its intensity distribution will vary. Again, this effect can be readily observed as the twinkling of stars. In the atmosphere, wave-front distortion and diffraction happen continuously along the propagation path.

The strength of the turbulence itself is expressed with the refractive-index structure constant $\mathrm{C}_{\mathrm{n}}^{2}$. The modelling of the spectrum of the turbulence-cells requires an inner and outer scale of turbulence $\left(\mathrm{l}_{0}\right.$ and $\left.\mathrm{L}_{0}\right)$, which is approximately the size of the smallest and largest turbulence cells in the regarded atmospheric volume. Typically, $\mathrm{l}_{0}$ is in the range of millimeters while $\mathrm{L}_{0}$ is up to several meters, both values grow with height. $1_{0}$ has strong impact on the smallest structures in the intensity-pattern which is important for fade statistics as described below.

\footnotetext{
Author Contact: dirk.giggenbach@dlr.de

Dirk Giggenbach, Institute of Communications and Navigation,

German Aerospace Center (DLR), Site Oberpfaffenhofen, D-82234 Wessling, Germany
}

Copyright 2003 Society of Photo-Optical Instrumentation Engineers.

This paper was published in Proc. of SPIE Vol. 4976 and is made available as an electronic reprint with permission of SPIE. One print or electronic copy may be made for personal use only. Systematic or multiple reproduction, distribution to multiple locations via electronic or other means, duplication of any material in this paper for a fee or for commercial purposes, or modification of the content of the paper are prohibited. 


\section{MEASUREMENT-SETUP}

\subsection{Optical Link Setup}

The transmitter of the experiment consists of a highly coherent 50mW Nd-YAG-laser emitting CW at $1064 \mathrm{~nm}$. The gaussian transmit beam has approx. $13 \mathrm{mrad}$ divergence. The transmitter is situated $400 \mathrm{~m}$ south of the receiver $15 \mathrm{~m}$ above ground on top of a tower. The $400 \mathrm{~m}$-path is horizontal. On the first half it leads over a meadow and then over asphalt.

The scintillometer-receiver features three apertures of different diameters (12, 22, and 48mm) and a CCD-camera for beam-control. The apertures integrate the intensity over their areas. The incoming light is focused onto silicon photodetectors, transformed into photocurrent and amplified into a voltage which is proportional to the light's power on each aperture. Most of the background-light is blocked by $1064 \mathrm{~nm}$-interference-filters. The three signals from the receivers are sampled by ADCs and stored. The whole scintillometer size is much smaller than the $5 \mathrm{~m}$ beam diameter at the receiver, thus the overall beam wander at the receiver does not cause intensity fluctuations. Data is sampled every ten minutes for 1.024 seconds with a rate of 2000 samples/second resulting in a recorded bandwidth of $1 \mathrm{kHz}$.

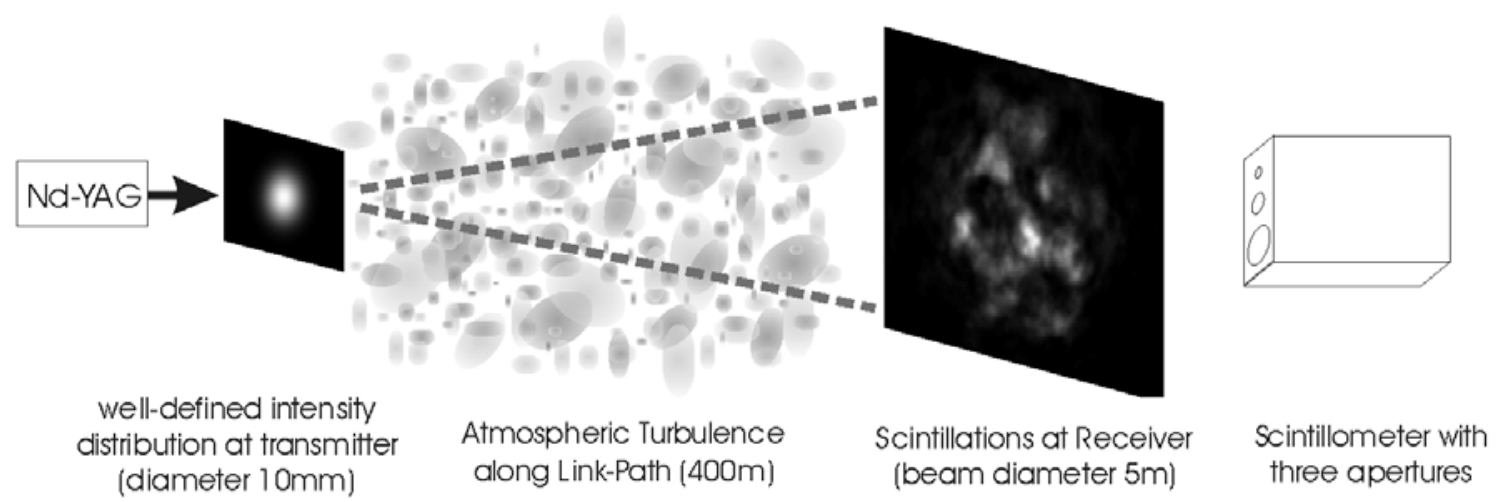

Figure 1: Optical link and scintillometer setup

\subsection{Weather Station}

Simultaneous to the Rx power vectors several meteorological values where measured at the Rx-site: Temperature, humidity, wind-velocity and -direction, rain-state (binary value), sunshine-state (binary value). The sunshine-sensor only reacted at very strong light irradiation, it can thus be assumed that the sun was often shining even without this sensor reacting. When values of the binary sensors are time-averaged this produces continuous values between 0 and 1 as reflected in the figures below.

\section{STATISTICAL EVALUATION}

The turbulence in this experiment can be assumed fairly equally distributed as the beam path is going horizontally over plane ground. Because the beam is diverging to about $5 \mathrm{~m}$ at the receivers, formulas have to regard a diverging gaussian beam, which in this case of near-axis reception comes close to a circular wave as seen from the receiver apertures.

The ideal receiver would have an infinitely small aperture to provide ideal scintillation data. This aperture of course would generate no signal, therefore it is sufficient to use an aperture which is smaller than the minimum structure sizes in the scintillation patterns. Even larger apertures can be used with the consideration of appropriate aperture-averaging factors in the formulas as stated below. 
In Fig. 2 one can see that there are no complete fades down to zero reception power in this transmission scenario (even after subtraction of background offsets). In longer propagation paths such zero points do occur in infinitely small spots as simulations and other experiments have shown. Zero points do require a longer path and/or stronger turbulence to emerge, see e.g. [4] and [5].

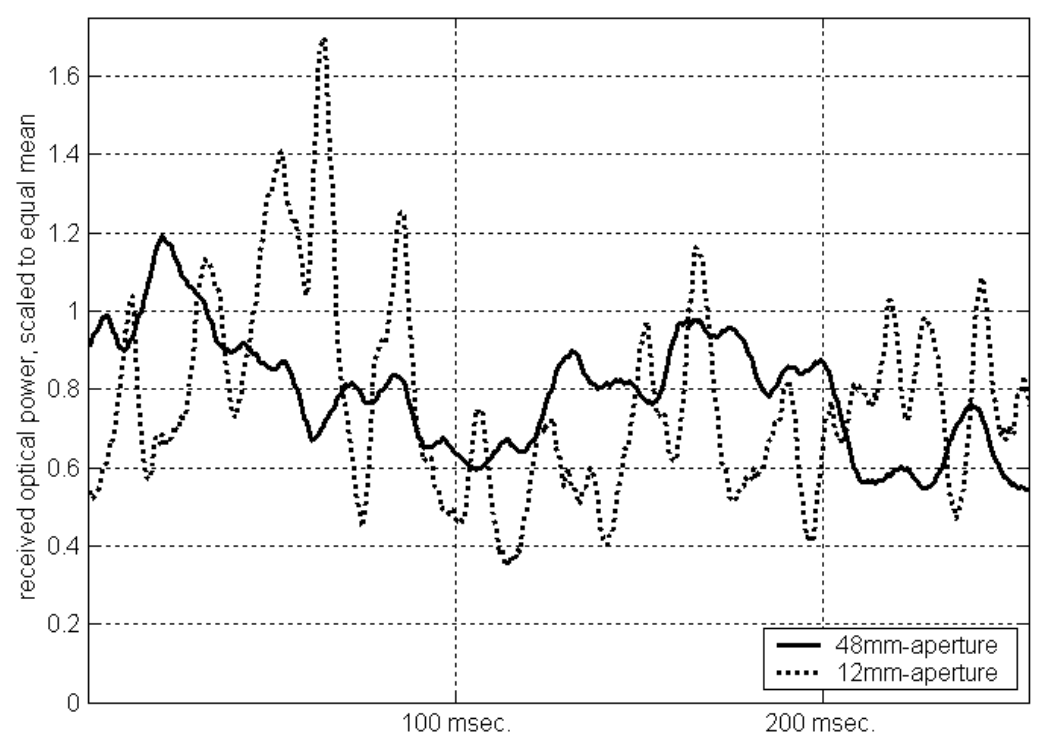

Figure 2: Typical normalized simultaneously received power fluctuations from the $48 \mathrm{~mm}$ - and the $12 \mathrm{~mm}$ - aperture, the scintillation index here was 0.12 .

Statistical values for the optical Rx-power are computed assuming weak-turbulence conditions and thus no saturation of scintillation effects are taken into account. The power scintillation index is the normalized variance of the received optical power as directly measured by the photodetectors behind each Rx-aperture:

$$
\text { power scintillation index }=\sigma_{\mathrm{D}}{ }^{2}=\frac{\left\langle P(t)^{2}\right\rangle}{\langle P(t)\rangle^{2}}-1
$$

Fades: Short-term Rx-power drops below a certain threshold value relative to the mean Rx-power are defined as fades. This threshold value must be chosen in order to produce reasonable statistical statements. Normally $3 \mathrm{~dB}$ from mean is used, here we chose $1.5 \mathrm{~dB}$ as $3 \mathrm{~dB}$ fades did not occur often enough.

Mean number of fades (NoF): The time spectral behaviour can be reflected by the average occurance of fades per seconds. Again we use the $1.5 \mathrm{~dB}$ limit. 
Aperture averaging factor: The aperture averaging factor is defined as the ratio of the Rx-power variance $\sigma_{\mathrm{D}}{ }^{2}$ with finite aperture of diameter " $D$ " to the variance $\sigma_{0}^{2}$ with infinitely small aperture:

$$
A=\frac{\sigma_{D}^{2}}{\sigma_{0}^{2}}<1
$$

This factor A needs to be considered when parameters derived from formulas containing the ideal $\sigma_{0}{ }^{2}$ are calculated from real data which always implies a finite aperture D.

A simple approximation for "A" assuming a spherical wave is given by [1]:

$$
A_{\text {spherical }}=\left[1+0.333 \cdot\left(\frac{\pi D^{2}}{2 L \lambda}\right)^{5 / 6}\right]^{-7 / 5}
$$

with

$$
\begin{aligned}
& \mathrm{L}=\text { path length }(400 \mathrm{~m}) \\
& \mathrm{D}=\mathrm{Rx} \text {-aperture diameter } \\
& \lambda=\text { optical wavelength }(1.064 \mu \mathrm{m})
\end{aligned}
$$

For our setup this results in $A[12,22,48 \mathrm{~mm}]=[0.778,0.546,0.217]$. This allows us to recalculate the sampled data to the "ideal" case with infinitely small apertures which then is the same as the variance of the intensity.

The effects of aperture averaging in terms of temporal low-pass filtering and variance-reduction are reflected in Fig.2: The smaller the aperture the stronger the Rx-power fluctuations and the broader the signal bandwidth.

Scintillation index: The normalized variance of the intensity $I$ is also called the scintillation-index " $\sigma_{I}^{2} "$ and can be calculated from the power variance with finite aperture:

$$
\sigma_{I}^{2}=\frac{\left\langle I^{2}\right\rangle}{\langle I\rangle^{2}}-1=\sigma_{D}^{2} /\langle P\rangle^{2} \cdot A(D)
$$

The measured scintillation-index value can be compared with the one derived analytically under weak turbulence assumptions (Rytov-conditions, [1]) for a spherical wave

$$
\sigma_{I-s}^{2}(L)=0,49 \cdot C_{n}^{2} \cdot k^{7 / 6} \cdot L^{11 / 6}
$$

In (5) $\mathrm{C}_{\mathrm{n}}{ }^{2}$ is the refraction-index structure parameter in $\mathrm{m}^{-2 / 3}, \mathrm{k}$ is the wavenumber $(2 \pi / \lambda)$ and $\mathrm{L}$ the propagation distance in meters. With a typical near-ground value for $\mathrm{C}_{\mathrm{n}}^{2}$ of $10^{-13} \mathrm{~m}^{-2 / 3}$ we do expect scintillation indices around 0.02 .

Further turbulence parameters like the mean turbulence structure parameter $C_{n}{ }^{2}$ or the atmospheric coherence length could be deduced from the received power vectors. Here we limit evaluation to the statistics of the received optical power. 


\section{VARIATIONS OF RX-POWER PARAMETERS}

\subsection{Diurnal variations of statistical parameters}

The following plots show comparisons of the daily parameter variations with three different meteorological situations.

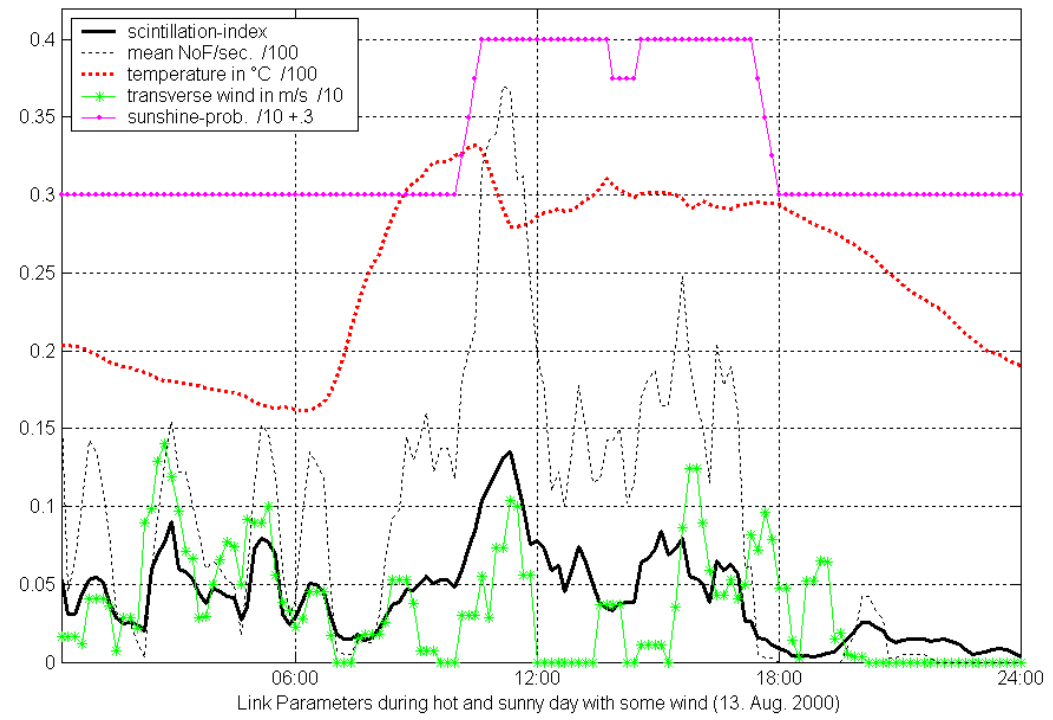

Figure 3: Link- and meteorological parameters on a hot and sunny day (NoF for $12 \mathrm{~mm}$ aperture)

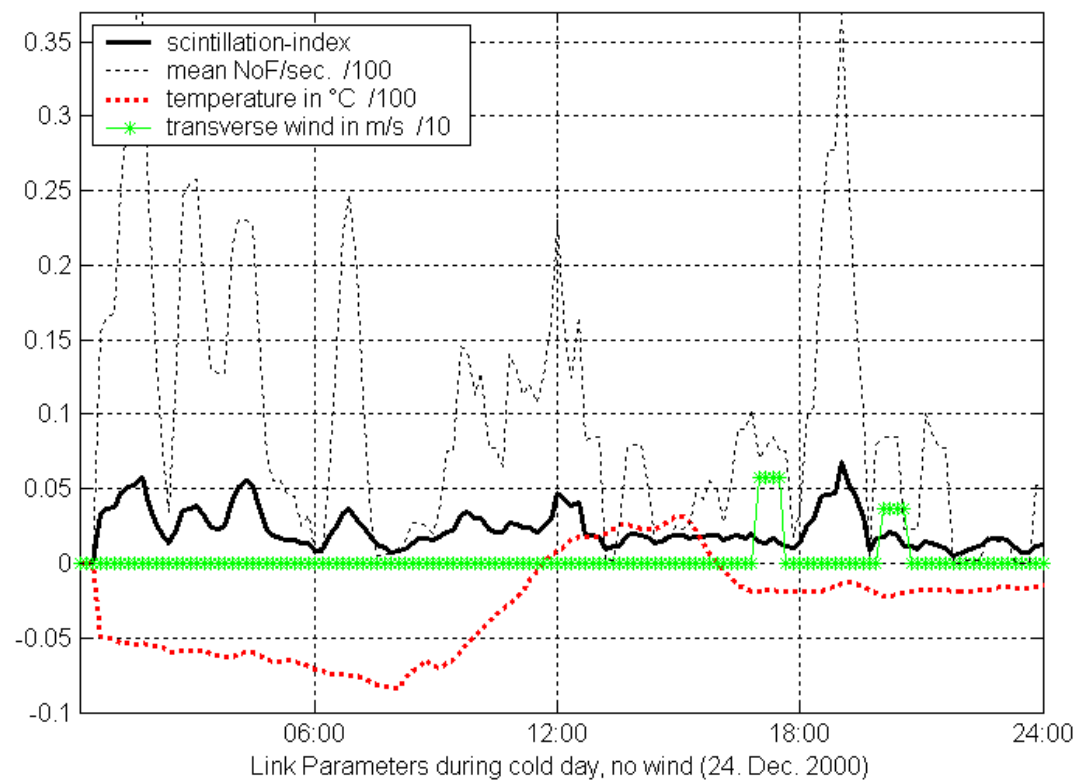

Figure 4: Link parameters on a cold and calm day (NoF for $12 \mathrm{~mm}$ aperture) 


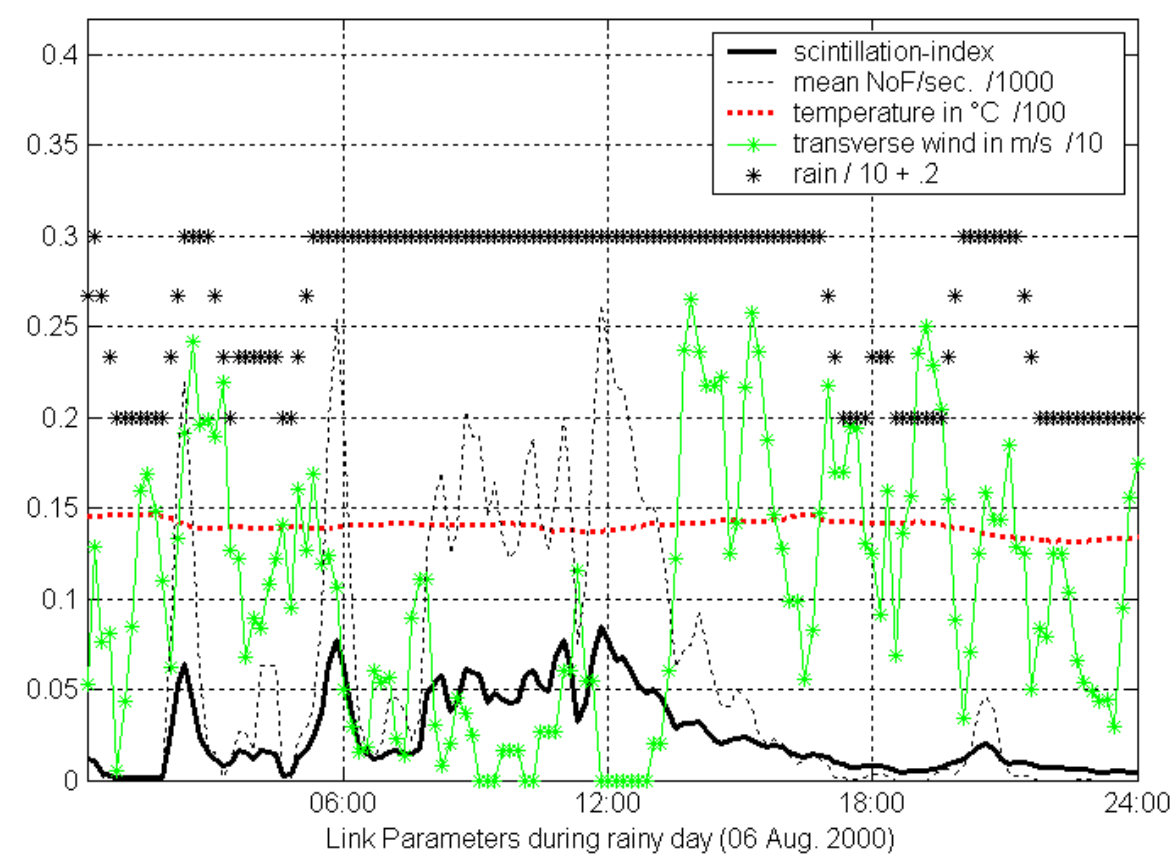

Figure 5: Link parameters on a rainy and windy day (NoF for $12 \mathrm{~mm}$ aperture)

Discussion: Under very cold conditions the mean scintillation-index drops to roughly half the values on hot days. A certain dependence of direct sun-heating compared to overcast weather situations is identifiable. The transversal wind component also seems to affect the signal bandwidth (i.e. mean number of fades, NoF) as seen in Fig.3, though as can be seen in Fig.4 the NoF must also be impacted by other effects as large values do occur even with no measured wind, one explanation might be insufficient wind recording as stated in section 5. Rain does also increase frequency of fades heavily as can be seen in Fig.5. On overcast days with constant temperature (and thus quite stable conditions) rain seems to have an increasing effect on the scintillation index and especially on the number of fades as can be seen in fig. 5 which shows very low scintillation and NoF during rain-breaks in contrast to times with rain.

\subsection{Seasonal variations of statistical parameters}

To make seasonal variations clearly visible it is necessary to take averages of several days. To avoid the influence of the strong diurnal turbulence variations it is preferable to state values each only for a certain time of day.

Due to necessary changes in the hardware, some parameters changed during the measurement period, thus preventing successional evaluation of some parameters over the whole experiment time. Some more dubious data which was due to non-meteorological link disturbances also had to be removed.

The next figure shows mean temperatures at noon together with the scintillation index. 


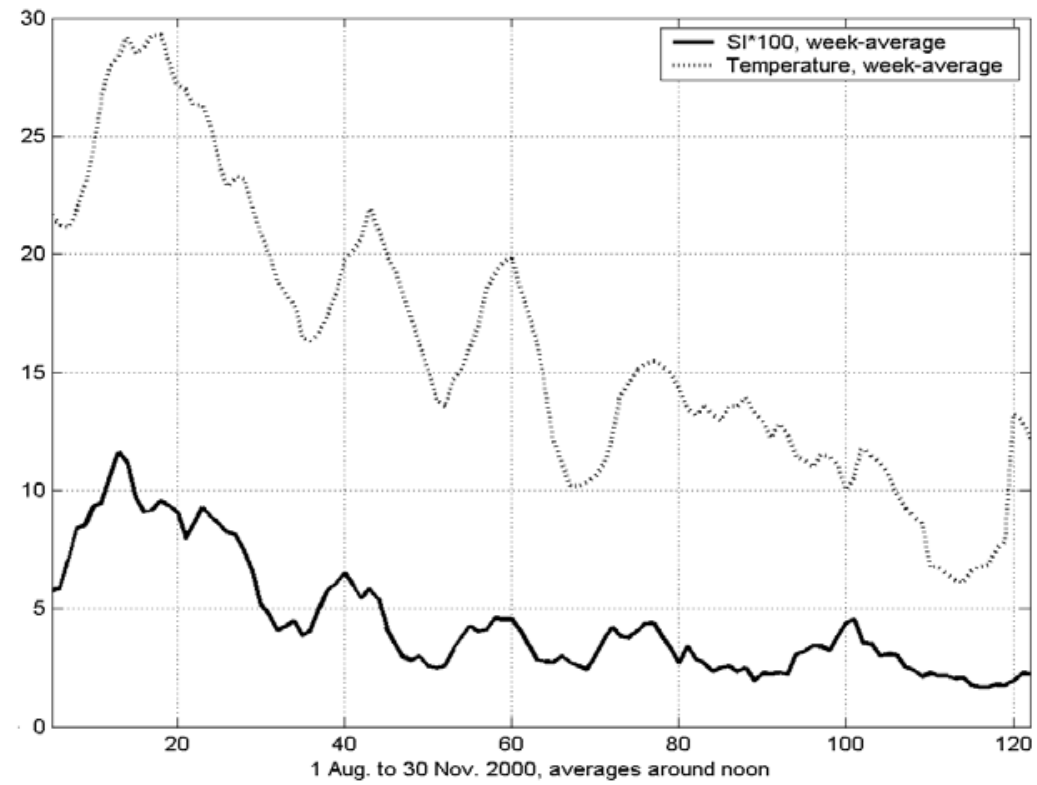

Figure 6: Correlation of week-averaged SI with week-averaged temperature from August to November between 12:00 and 01:00 pm. Abscissa is in days.

These week-averages of temperature and scintillation-index show a normalized cross correlation coefficient of up to 0.9. The situation at other times of day does not show such strong correlation, especially temperature seems to have no indication at all during night. This suggests that the effect might rather be one of sunshine irradiation than of temperature. The next plot therefore shows scintillation index and mean sunshine-probability during summer in the afternoon.

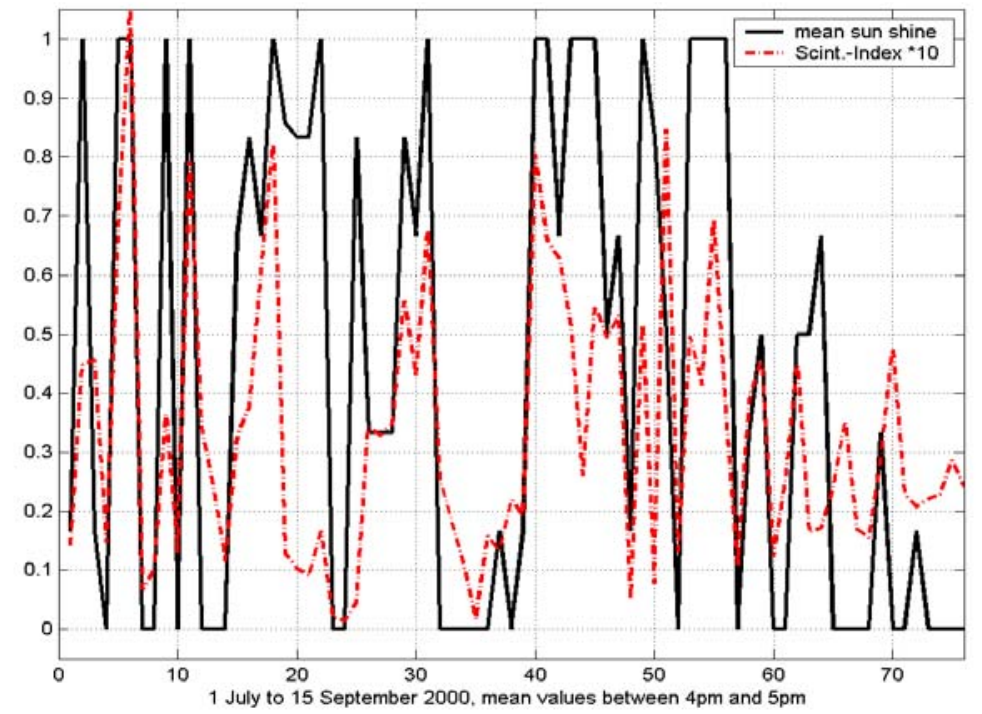

Figure 7: Correlation of sunshine and magnitude of scintillation-index during summer in the afternoon

The normalized cross-covariance index of sunshine and scintillation-index during summer from 12:00 to 17:00 lies between 0.60 and 0.68 . Similar correlations could be observed between sunshine and the mean number of fades and the probability of fades. These observations - maybe connected with temperature measurements - allow a qualitative prediction of the scintillation index. 


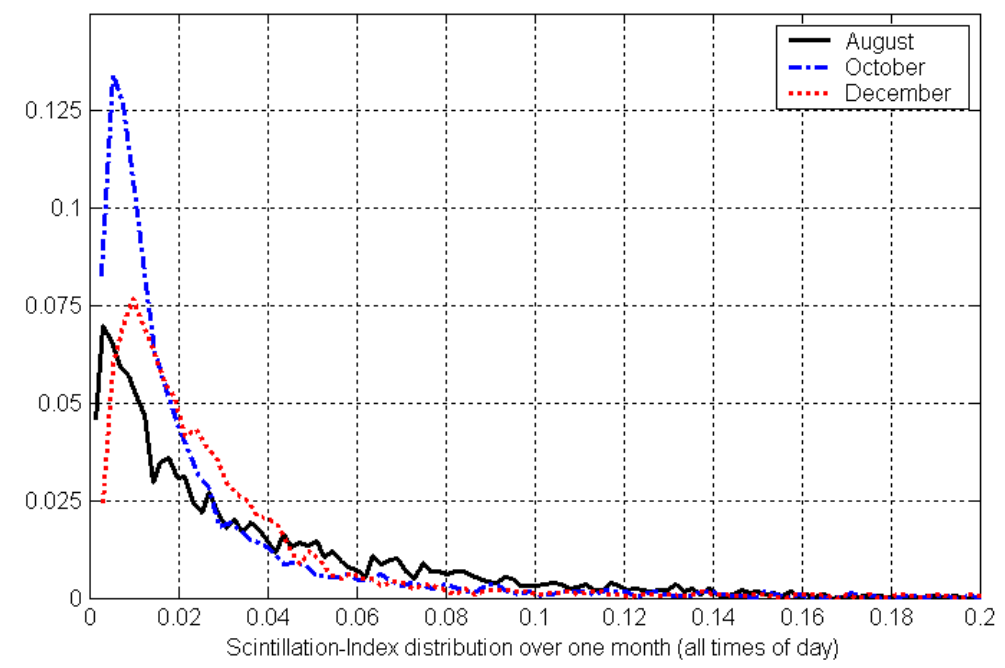

Figure 8: Scintillation-index distribution over all measurements (irrespective of time of day) in August (mean: 0.037), October (mean: 0.028), and December (mean: 0.035)

The whole-month distribution of the scintillation index (Fig.8) shows lower values and also lower variance of the scintillation index in autumn which might correspond to the more frequent overcast sky and thus calmer turbulence, while August and December feature a broader distribution which corresponds to a wider range of the turbulence-state during the more extreme times of the year.

\section{RESULTS}

\subsection{Seasonal variations}

Unlike with the diurnal variations and meteorological boundary conditions, scintillations have only weak connection with the season. While in winter the turbulence seems mostly smaller and less varying than during the warmer season, the diurnal and short-term meteorological variations dominate over the seasonal effects by far. During summer the turbulence activity seems to be strongly related to sunshine and thus direct boundary-layer warming.

The span of the path-averaged refraction-index structure constant $\mathrm{C}_{\mathrm{n}}{ }^{2}$ based on the measured scintillation index and calculated with (5) lies between $10^{-12} \mathrm{~m}^{-2 / 3}$ and $5 \cdot 10^{-15} \mathrm{~m}^{-2 / 3}$ which agrees with other observations like those presented in [3].

\subsection{Diurnal variations}

While in the late afternoon and evening until midnight the atmospheric conditions usually can be regarded as stabilized, scintillation rises with atmospheric warming during the morning until 11 o'clock, where it peaks and then drops to an intermediate level. Smaller peaks can be observed around dawn and sunset. The situation during the second half of the night is unclear, frequently turbulence activity can be observed that might be related to strong winds. Also, during some days a correlation of the number of fades with the temperature-gradient and with orthogonal wind velocity could be observed, this does not hold for the majority of observed days. Nonetheless from general geodetic and meteorological observations it could be expected that for a longer and more elevated beam path the temperature gradient is a good indicator for destabilizing changes in the atmospheric boundary layer.

Number of fades and scintillations are increased during rain. To our surprise only a vague relation between duration of fades and transverse wind velocity could be observed. One reason might be the fact that the weather station can only provide data at the receiver location, this certainly does not represented the wind impact over the whole link path sufficiently. Another reason might be that the frozen-turbulence assumption - that lateral wind-movements dominate over convective inner cell-movements with at least one order of magnitude - does not hold in this near-ground scenario (15m above ground). 
We can summarize that the sunshine-state (clear sky with direct sun heating in contrast to overcast sky), airtemperature, and wind velocity are the predominant indicators for turbulence in our scenario during daytime. This corresponds also to other experimental observations over longer paths ([4] and [5]). For night time no dominant dependencies could be found except for rain.

\section{REFERENCES}

[1]

L. C. Andrews, R. L. Phillips:

"Laser Beam Propagation through Random Media"

SPIE Press, Bellingham (WA), 1998

[2]

V. I. Tatarskii:

"The Effects of the Turbulent Atmosphere on Wave Propagation"

Israel Program for Scientific Translations, Jerusalem 1971

[3]

J.W. Strohbehn (editor):

"Laser Beam Propagation in the Atmosphere"

Springer Verlag 1978

[4]

D. Giggenbach, F. David, et al.:

"Measurements at a $61 \mathrm{~km}$ near-ground optical transmission channel",

Proceedings of SPIE Vol. 4635, 2002

[5]

F. David, D. Giggenbach, et al.:

"Overview of the FASOLT Experiment and Final Results",

Free-Space Laser Communications Technologies XV,

in Proceedings of the SPIE Vol. 4975, 2003 